\title{
Uma Análise do Uso de Avaliações por Pares e Rubricas na Promoção de Interação entre Alunos em uma Disciplina de Graduação em Engenharia de Computação
}

\author{
Tiago de Oliveira - Unifesp - tiago.oliveira@unifesp.br \\ Denise Stringhini - Unifesp - dstringhini@unifesp.br \\ José Jailson Santos Craibas - Unifesp - jose.craibas@unifesp.br \\ Deborah Godoy Martins Corrêa - Unifesp - deborah.correa@unifesp.br
}

Resumo: Este artigo apresenta uma análise sobre o uso da combinação das técnicas de avaliação por pares e de rubricas na promoção de interação entre alunos em práticas de ensino baseadas em problemas numa disciplina de graduação em Engenharia de Computação. A metodologia de ensino adotada na disciplina se estrutura no preenchimento de fichas de avaliação pelos alunos de acordo com rubricas previamente disponibilizadas pelo instrutor. Essa metodologia foi aplicada para uma turma de 25 alunos, tendo sido analisados os comentários produzidos nas fichas de avaliação e os trabalhos desenvolvidos ao longo do semestre. Como resultado, conclui-se que o emprego dessas técnicas tem mostrado a efetivação de um ambiente educacional favorável à interação, verificando-se, inclusive, a implementação de itens não obrigatórios nos trabalhos desenvolvidos pelos alunos.

Palavras-chave: avaliação por pares, rubrica, aprendizado baseado em problemas.

\section{An Analysis of Online Peer Assessments and Scoring Rubrics Used to Promote Student Interaction in a Computer Engineering Undergraduate Course}

\begin{abstract}
In this article, it is analyzed the use of online peer assessments and scoring rubrics techniques with the objective of promoting interaction between students in problem-based teaching practices in a Computer Engineering undergraduate course. The teaching methodology adopted in the course is structured in the completion of assessment sheets by the students according to the rubrics previously made available by the instructor. This methodology was applied to a group of 25 students and the comments produced in the assessment sheets and the works developed during the semester were analyzed. As a result, it is concluded that the use of these techniques has shown the effectiveness of an educational environment favorable to the interaction between students, in addition to the implementation of optional items in the works developed.
\end{abstract}

Keywords: online peer assessment, scoring rubric, problem based learning.

\section{Introdução}

A prática de ensino denominada aprendizado baseado em problemas (PBL) foi inicialmente proposta na Escola de Medicina da MacMaster University, no Canadá (Barrows e Tamblyn, 1980; Sales et al., 2013) e o seu elemento-chave reside no fato de que o problema comanda o processo de aprendizagem do aluno. A partir de uma determinada situação-problema, o aluno é incentivado a construir seu próprio aprendizado, tomando decisões, investigando, refletindo e estudando os conceitos teóricos e práticos necessários para a realização de sua solução para a situação-problema delineada. Dependendo das unidades curriculares onde essa prática é aplicada, as situações-problema podem variar, podendo-se citar, como exemplos, análises de casos clínicos, simulações de sistemas, desenvolvimento de produtos tecnológicos e realizações de projetos de pesquisa ou de engenharia. 
Dentro deste contexto, neste artigo, busca-se avaliar a utilização combinada das técnicas de avaliações por pares e rubricas em práticas de ensino baseadas em problemas em relação à promoção da interação entre os alunos numa disciplina de Engenharia de Computação.

Na sequência, na seção 2, são introduzidos os conceitos de avaliação por pares e de rubricas como métodos de ensino-aprendizagem. Na seção 3, define-se a metodologia de ensino-aprendizagem no contexto PBL que foi utilizada buscando-se promover a interação entre os alunos. Na seção 4, apresentam-se os resultados obtidos com a aplicação dessa metodologia em uma disciplina de Laboratório de Arquitetura e Organização de Computadores de um curso de Engenharia de Computação. Por fim, na seção 5, encontram-se as considerações finais sobre a análise realizada e sobre a metodologia de ensino-aprendizagem aplicada.

\section{Fundamentação Teórica}

\subsection{Avaliações por Pares}

A avaliação por pares (Tenório et al., 2016) é uma alternativa à avaliação tradicional, buscando unir a etapa de avaliação aos processos de ensino e de aprendizagem do aluno, permitindo-o o acompanhamento, a avaliação e a regulação da sua própria aprendizagem.

A avaliação por pares pode ser definida como um cenário em que alunos atuam na revisão de trabalhos de outros alunos de mesmo nível por meio de um acordo ou conjunto de critérios definidos pelo professor (Trahasch, 2004; Topping, 2003). Enquanto avaliados, seus trabalhos são revisados pelos colegas e, enquanto avaliadores, realizam retorno sobre os trabalhos de seus colegas. Todo esse processo pode possibilitar que os alunos pensem mais profundamente sobre determinado assunto, conduzindo-os a uma aprendizagem mais significativa (Trahasch, 2004), onde podem refletir sobre os objetivos alcançados e adotar medidas junto ao professor responsável pela disciplina para a superação de dificuldades em direção aos objetivos desejados. Além disso, quando um aluno avalia trabalhos desenvolvidos por seus colegas, indiretamente, ele está aprendendo a se autoavaliar e a ajustar o seu próprio trabalho em direção aos objetivos estabelecidos, desenvolvendo, com isso, senso crítico, capacidade de análise e de reflexão sobre os objetivos desejados e alcançados. Assim, a avaliação passa a ser uma compilação de ações e intenções, centrada no aluno e não no professor.

A avaliação por pares tem sido aplicada com sucesso no ensino superior em áreas diversificadas, podendo-se citar, como exemplos, o trabalho publicado em Wang et al. (2012) sobre o uso dessa prática de ensino na área de linguagens de programação em Computação; o trabalho publicado em Asikainen et al. (2014) na área de Biociência; o trabalho publicado em $\mathrm{Ng}$ (2014) para a formação de professores; o trabalho publicado em Garousi (2010) numa disciplina relacionada à engenharia e; os trabalhos nacionais publicados em Silva e Moreira (2003), Ugulino et al. (2009) e Barbosa e Nelson (2016) relacionados à aplicação da avaliação por pares em cursos de graduação e pós-graduação em, respectivamente, disciplinas de computação, uma disciplina de Metodologia de Pesquisa Científica e em disciplinas de Modelagem e Construção de Aplicações Web.

Como observa-se nos exemplos citados anteriormente, se corretamente aplicada, a avaliação por pares pode enriquecer a aprendizagem por meio de um maior envolvimento dos alunos em atividades avaliativas, obtendo-se uma participação ativa de todos os atores, os quais, em conjunto, contribuem para que os objetivos estabelecidos sejam alcançados. 


\subsection{Rubricas}

A utilização dessa prática de ensino pode orientar os alunos a atingirem níveis de qualidade desejáveis, pois facilita a comunicação e a interação entre professores e alunos e entre grupos de alunos na definição dos objetivos curriculares de aprendizagem a serem alcançados (Piedra et al., 2010). Uma definição comumente utilizada para rubrica refere-se a um documento que descreve a articulação das expectativas de uma determinada tarefa, listando-se os critérios ou quesitos que devem ser analisados e descrevendo-se os níveis de qualidade desejáveis em relação a cada um dos critérios discriminados (Reddy e Andrade, 2010). Essas tarefas podem corresponder aos mais variados tipos de objetos ou artefatos de aprendizagem, tais como, produtos ou protótipos, apresentações orais, mapas de conceitos, artigos, pôsteres e exames.

Esta prática de ensino pode ser incluída em dois contextos educacionais, quais sejam: na avaliação dos alunos e nos processos de ensino e de aprendizagem. No primeiro contexto, os artefatos produzidos pelos alunos podem ser avaliados e as rubricas podem ser utilizadas para aferição e estabelecimento de uma determinada nota ou conceito final relacionados à qualidade de cada um ou de um conjunto desses artefatos produzidos (Campbell, 2005). No segundo contexto, ao se disponibilizar antecipadamente critérios bem definidos e delineados da avaliação e os seus níveis de desempenho desejáveis, as rubricas podem ser utilizadas para facilitar a produção de retornos formativos (feedbacks) sobre o progresso dos alunos (Andrade, 2000), além de servirem como guia do processo de aprendizagem, permitindo a sua utilização como parâmetro no desenvolvimento, revisão e julgamento de trabalhos (Huba e Freed, 2000).

Como exemplos de rubricas no ensino superior podem-se citar o desenvolvimento de um conjunto de ferramentas de software para facilitar a aplicação no ensino do quadro europeu de qualificações (European Qualifications Framework - EQF) relacionado ao processo de Bolonha (Florian-Gaviria et al., 2013); a construção de rubricas para ser utilizadas na avaliação de monografias de graduação de um curso de Enfermagem (González-Chordá et al., 2016); a aplicação de rubricas para avaliação e para o aprendizado em cursos da área de economia (Mcgoldrick e Peterson, 2013); e os trabalhos nacionais sobre a avaliação de um Web fórum por meio de rubricas num curso de graduação em Ciências Biológicas (Ferreira e Silva, 2010) e sobre a avaliação qualitativa de estudantes em cursos de Computação e Pedagogia (Lobato et al., 2009).

Como pode ser constatado nos exemplos apresentados no parágrafo anterior, as rubricas têm sido utilizadas com sucesso tanto na aferição de desempenho ou competências quanto nos processos de ensino e de aprendizagem dos alunos, facilitando o estabelecimento e divulgação de critérios, metas e objetivos a serem cumpridos.

\section{Metodologia de Ensino-Aprendizagem Adotada na Disciplina}

Na Universidade Federal de São Paulo - Unifesp, o ensino de Arquitetura e Organização de Computadores está inserido num currículo de curso de Engenharia de Computação diferenciado, sendo estruturado em uma abordagem prática e sistêmica que busca, além de integrar teoria e prática e também hardware e software, reduzir a visão fragmentada dos alunos de um sistema computacional complexo. Mais detalhes sobre essa estrutura curricular diferenciada da Unifesp podem ser encontrados no projeto pedagógico do curso $^{1}$ e no artigo publicado em Oliveira et al. (2017).

${ }^{1} \mathrm{O}$ projeto pedagógico do curso de graduação em Engenharia de Computação da Unifesp foi aprovado em 2015, disponível em: http://www.unifesp.br/campus/sjc/o-curso-engcom/projeto-pedagogico-do-curso.html, acesso em 03 set. 2018. 
Dentro dessa estrutura encontra-se a disciplina de Laboratório de Arquitetura e Organização de Computadores, a qual ocorre em 4 horas semanais durante vinte semanas para turmas de 25 alunos, onde cada aluno deve desenvolver em linguagem de descrição Verilog sua própria plataforma de hardware, composta por processador, memória e sistema de entrada/saída utilizando um kit didático específico.

Para o cumprimento desses objetivos, a metodologia de ensino foi subdividida em quatro pontos de checagem (PCs), sendo eles: no primeiro ponto de checagem (PC1), os alunos são estimulados a pesquisar o funcionamento e arquitetura interna de vários processadores da literatura e a começarem a definir o conjunto de instruções (ISA) que será suportado pela sua plataforma de hardware; no segundo ponto de checagem (PC2), o aluno deve realizar um detalhamento maior do esboço da arquitetura interna e projetar em Verilog a unidade de processamento da plataforma de hardware; no terceiro ponto de checagem (PC3), o aluno deve realizar a especificação em Verilog da unidade de controle da plataforma de hardware proposta e, por fim, no quarto e último ponto de checagem (PC4), o aluno deve trabalhar na integração de todos os módulos produzidos, interligando a unidade de processamento e de controle da plataforma de hardware.

Em cada um dos quatro pontos de checagem, um relatório técnico deve ser confeccionado por aluno e um processo de avaliação por pares deverá ser realizado. Quanto ao processo de avaliação por pares, são atribuídos seis relatórios técnicos por aluno. A cada novo PC, atribuem-se relatórios técnicos diferentes, buscando-se evitar a repetição de alunos em relação aos pontos de checagem anteriores para um determinado avaliador. Sendo assim, no final da disciplina, um aluno terá analisado 24 relatórios técnicos diferentes, permitindo-o ter tido contato com os projetos que estão sendo desenvolvidos por toda a turma, já que a disciplina possui no máximo 25 alunos no total.

Especificamente sobre os relatórios técnicos que serão elaborados pelos alunos, a metodologia de ensino estrutura-se em avaliações por pares que serão utilizadas para (1) permitir aos alunos acompanharem os trabalhos que estão sendo desenvolvidos por outros alunos e observarem as diversas estratégias adotadas para a resolução das situaçõesproblemas, os caminhos que estão sendo seguidos, as ferramentas que estão sendo utilizadas e as dificuldades que estão sendo encontradas pelos seus pares; (2) possibilitar que os alunos interajam entre si, propondo novos caminhos, alternativas e relatando seus pontos de vistas sobre os trabalhos que estão sendo desenvolvidos pelos seus pares, buscando-se, com isso, uma aprendizagem mais significativa; (3) desenvolver nos alunos senso crítico e capacidade de auto-avaliação e auto-reflexão ao analisarem os trabalhos que estão sendo desenvolvidos pelos seus pares e, por consequência, seus próprios trabalhos e; (4) construir com informações alimentadas pelos pares um ambiente favorável que incentive ajustes e adequações visando melhorias nos artefatos finais a serem produzidos na resolução de cada situação-problema estabelecida e em direção ao cumprimento dos objetivos da disciplina.

Basicamente, uma ficha de avaliação é providenciada pelo instrutor da disciplina para ser utilizada pelos alunos durante a avaliação por pares dos relatórios técnicos entregues. Para essa ficha de avaliação, foram especialmente definidas e elaboradas as rubricas discriminadas na Tabela 1 para serem empregadas na aplicação da metodologia de ensino. Na Tabela 1, observa-se que a ficha de avaliação do relatório estrutura-se em nove dimensões, quais sejam: cabeçalho, introdução, objetivos, fundamentação teórica, desenvolvimento do trabalho, resultados obtidos e discussão, considerações finais, referências bibliográficas e aspectos gerais. Para cada dimensão existe um conjunto de perguntas e uma seção final de comentários. Por sua vez, para cada pergunta existe uma escala ou estratificação de pontuação, devendo os alunos assinalar uma dentre as estratificações disponíveis para cada pergunta de acordo com a qualidade das respostas encontradas no relatório técnico que está em análise. Além disso, no final de cada 
dimensão, é de extrema importância que os alunos preencham a seção de comentários, descrevendo os pontos fortes e os pontos fracos da dimensão em análise, explicitando possíveis melhorias ou caminhos a serem percorridos.

Logo após a disponibilização da ficha de avaliação, o instrutor deve buscar demonstrar aos alunos como eles deverão proceder como avaliadores em relação aos relatórios técnicos, estabelecendo de forma clara e sistemática os critérios definidos. Além disso, no final de cada PC, o instrutor precisa fazer uma compilação dos relatórios técnicos produzidos e das fichas de avaliação que foram preenchidas, devendo verificar e informar aos alunos as suas corretudes e adequações necessárias em relação aos objetivos definidos para o PC.

Tabela 1 - Ficha de avaliação elaborada para o relatório técnico

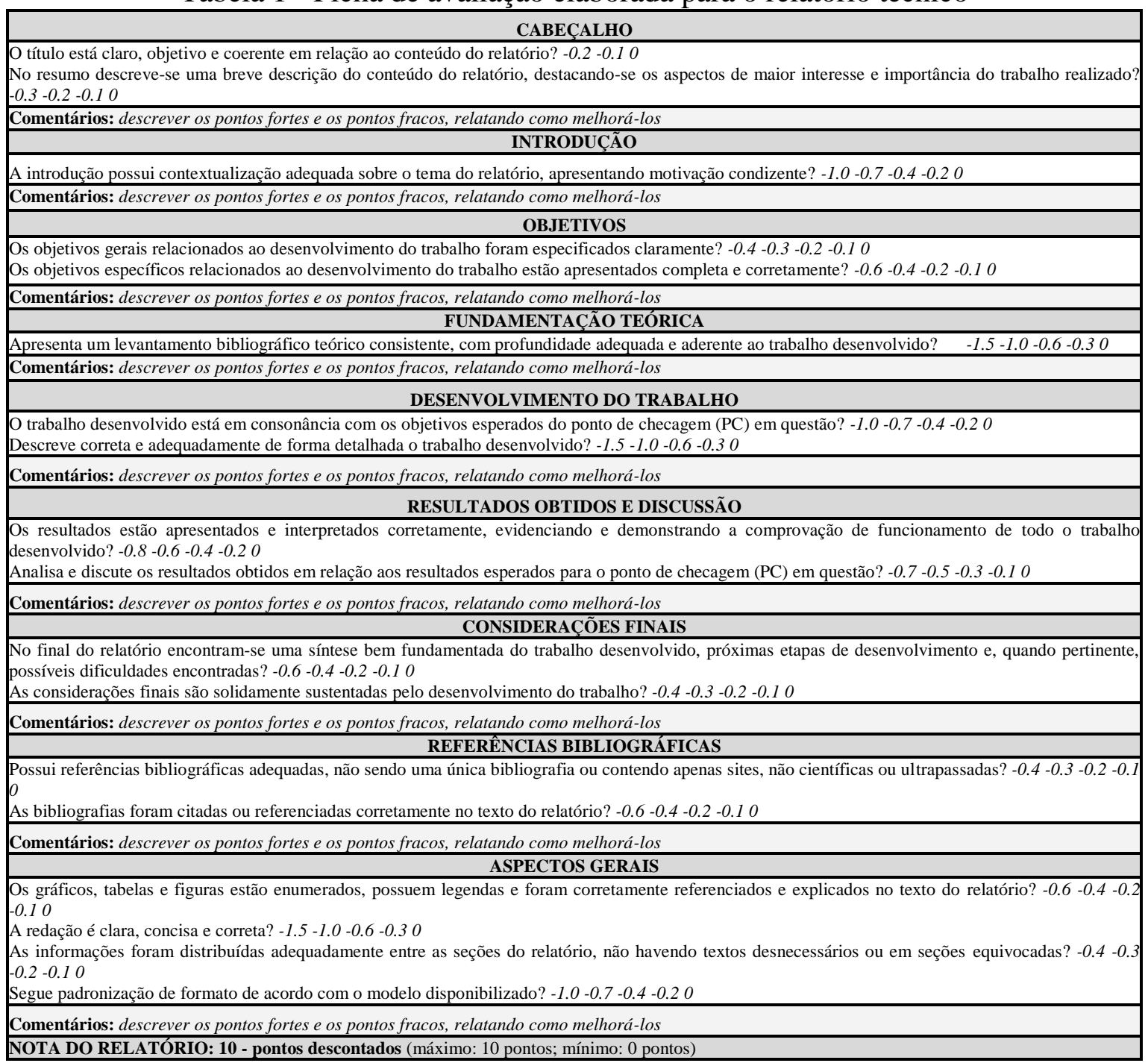

Fonte: os autores.

Por fim, o ambiente virtual de aprendizagem Moodle $^{2}$ foi utilizado para suportar a aplicação da metodologia de ensino, tendo sido organizado para permitir a realização, o mapeamento e a submissão das atividades propostas em cada ponto de checagem. $\mathrm{O}$ ambiente virtual Moodle foi escolhido por ser a plataforma institucional adotada na

2 O Moodle (Modular Object-Oriented Dynamic Learning Environment) é uma plataforma computacional de código aberto utilizado como apoio à aprendizagem do aluno. Mais detalhes sobre essa plataforma podem ser encontrados no endereço eletrônico https://moodle.org, acesso em 03 set. 2018. 
Unifesp, facilitando a configuração de contas, gerenciamento de conteúdo, agendamento e programação de atividades, distribuição de mensagens, aplicação das rubricas e realização da avaliação por pares. Além disso, os alunos já estão familiarizados com essa plataforma por a terem utilizado em outras unidades curriculares.

\section{Resultados Obtidos e Discussão}

Nesta seção, apresentam-se uma análise dos comentários produzidos pelos alunos durante o processo de avaliação por pares e uma análise dos trabalhos desenvolvidos durante a realização da disciplina. Para a coleta dos dados referente à análise dos comentários, foram utilizadas as fichas de avaliação preenchidas pelos alunos de acordo com a Tabela 1 e submetidas no Moodle quando da realização da atividade de avaliação por pares. Para a coleta dos dados sobre os trabalhos desenvolvidos, foram utilizadas as fichas de avaliação e os artefatos produzidos pelos alunos e submetidos no Moodle para avaliação. Os alunos que participaram dessas análises são majoritariamente do quarto e sexto termos do curso de Engenharia de Computação, com idades entre 19 e 26 anos e com aproximadamente $82 \%$ sendo do sexo masculino e $18 \%$ do sexo feminino.

\subsection{Análise dos Comentários Produzidos}

Para uma análise do uso da avaliação por pares na metodologia de ensino adotada e levando em consideração os conceitos apresentados em Gielen e Wever (2015) e em Narciss (2008), idealizou-se uma taxonomia ou diagrama de representação/codificação para os comentários produzidos pelos alunos no preenchimento das fichas de avaliação dos relatórios técnicos. Na Figura 1 apresenta-se a taxonomia definida para os comentários provenientes das fichas de avaliação, em que cada comentário pode ser classificado de acordo com o seu tipo e com o seu enfoque.

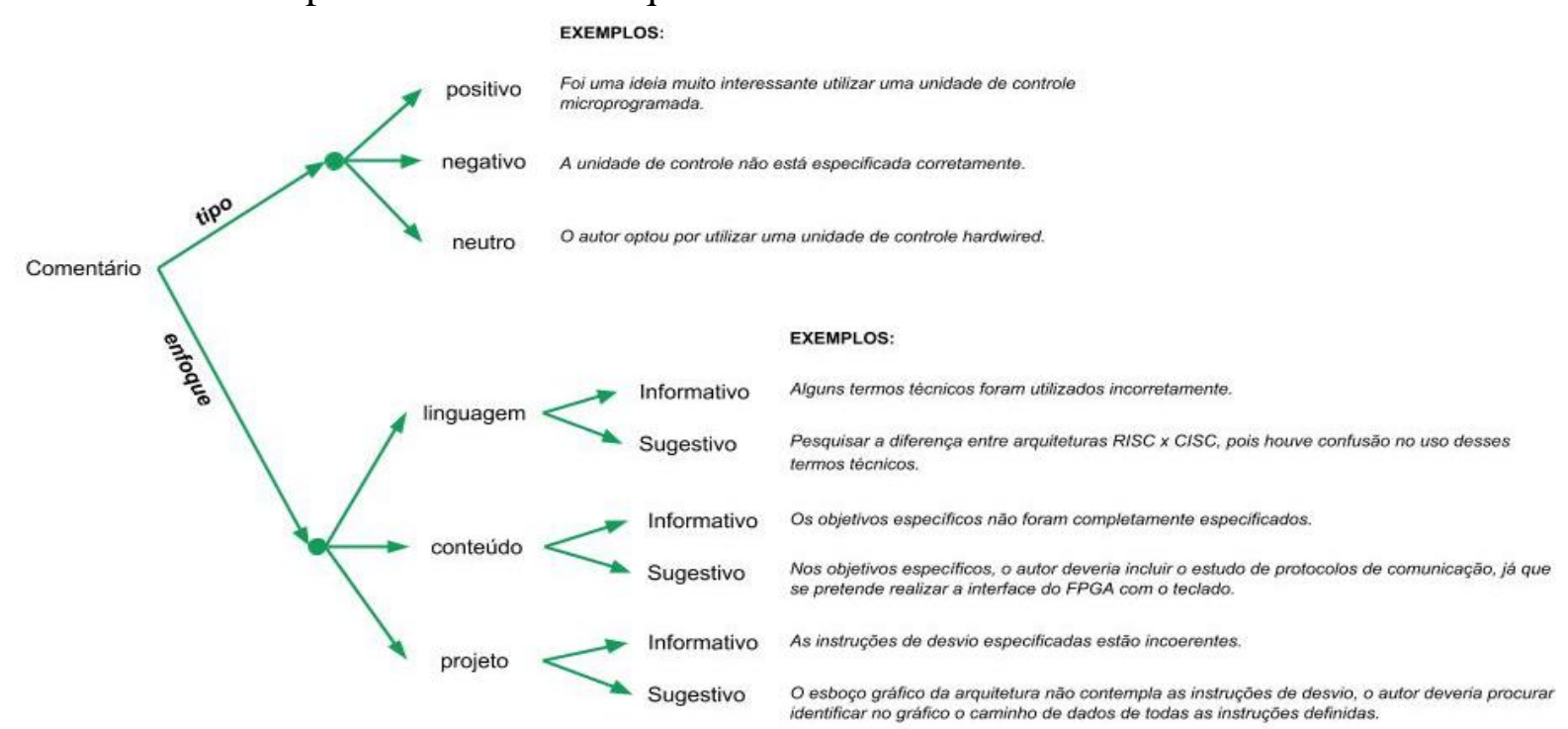

Fonte: os autores.

Figura 1 - Definição de uma taxonomia para os comentários das fichas de avaliação do relatório técnico

Em relação ao tipo, um determinado comentário pode ser classificado como positivo, negativo ou neutro, como exemplificado na Figura 1. Um comentário positivo implica uma sentença de concordância ou expressa uma avaliação positiva sobre uma determinada declaração ou sobre um determinado assunto/tópico. Um comentário 
negativo implica uma discordância ou expressa uma avaliação negativa, enquanto em um comentário neutro não há uma avaliação positiva nem negativa.

Por sua vez, além do tipo, um comentário também pode ser classificado quanto ao seu enfoque, podendo estar relacionado à linguagem, ao conteúdo ou ao projeto, como exemplificado no diagrama da Figura 1. Quanto à linguagem, o comentário realizado refere-se às características da língua portuguesa, como conjunção verbal, tradução, gramática, pronome, ortografia, construção semântica e layout. Quanto ao conteúdo, o comentário realizado refere-se à estrutura lógica dos conteúdos abordados ou das informações descritas e sua adequação com o que está sendo executado. Por fim, quanto ao projeto, o comentário realizado foca em questões sobre o desenvolvimento ou a especificação do projeto que está sendo realizado. Dentro da classificação quanto ao seu enfoque, o comentário pode ainda ser subclassificado como informativo ou sugestivo. Um comentário informativo refere-se a uma sentença ou expressão de cunho apenas informativo, quando o avaliador procura informar, julgar, confirmar ou justificar as avaliações que estão sendo realizadas, sem prover, no entanto, subsídios ou auxílios para que o autor possa alterar ou adaptar o seu trabalho. Um comentário sugestivo, por sua vez, possui também o propósito de sugerir, aconselhar e auxiliar o autor, visando o desempenho futuro do trabalho que está sendo realizado.

Utilizando a taxonomia definida na Figura 1, foram computados todos os comentários negativos realizados pelos alunos nas fichas de avaliação, tendo em vista que, potencialmente, esse tipo de comentário possui um efeito mais transformador no desenvolvimento do trabalho e na confecção de relatórios. Na Figura 2 encontra-se o levantamento de dados referente aos comentários realizados pelos alunos durante o oferecimento da disciplina de Laboratório de Arquitetura e Organização de Computadores.

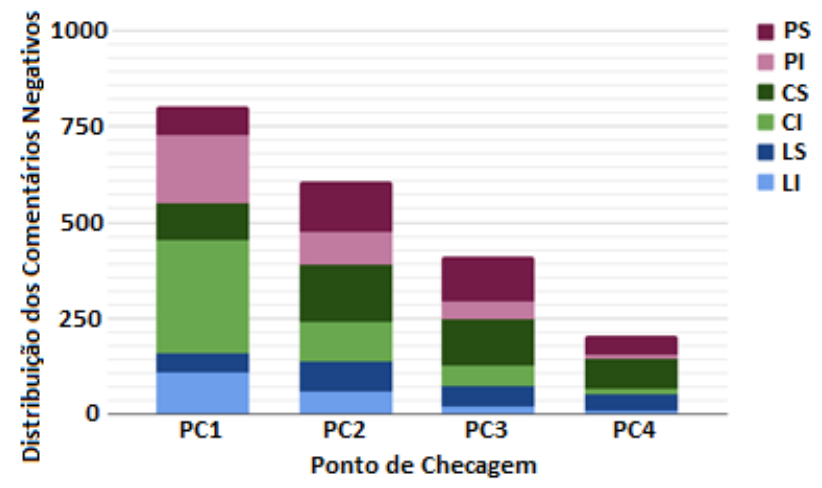

Legenda - PS: comentário Sugestivo relacionado ao Projeto; PI: comentário Informativo relacionado ao Projeto; CS: comentário Sugestivo relacionado ao Conteúdo; CI: comentário Informativo relacionado ao Conteúdo; LS: comentário Sugestivo relacionado à Linguagem e; LI: comentário Informativo relacionado à Linguagem.

Fonte: os autores.

Figura 2 - Distribuição dos comentários negativos produzidos pelos alunos no preenchimento das fichas de avaliação de acordo com a taxonomia definida

Em linhas gerais, a quantidade total de comentários negativos vai diminuindo entre a realização dos pontos de checagem. No PC1 foram produzidos 971 comentários negativos, no PC2 796 comentários, no PC3 601 comentários e no PC4 foram 395 comentários. Essa tendência de queda dos comentários negativos ao longo dos pontos de checagem é um indicativo de que a metodologia de ensino está contribuindo para o aprimoramento dos alunos quanto à linguagem de escrita, à estruturação de conteúdos e à especificação e o desenvolvimento de projetos.

Outro fato interessante refere-se à quantidade de comentários informativos e sugestivos produzidos. Note-se que, inicialmente no $\mathrm{PC} 1$, os alunos costumam produzir mais comentários informativos do que comentários sugestivos, como pode ser observado na Figura 2, onde aproximadamente $62 \%$ do total de comentários produzidos foram classificados como informativos. No entanto, essa relação tende a se inverter com a 
sequência dos próximos PCs, produzindo-se mais comentários sugestivos do que informativos: no PC4, aproximadamente $75 \%$ do total de comentários produzidos são classificados como sugestivos. Essa tendência de inversão é um reflexo direto da intervenção do instrutor da disciplina quando da demonstração de como os alunos deverão proceder como avaliadores em cada ficha de avaliação disponibilizada e do retorno realizado aos alunos após o término da avaliação por pares, buscando, a cada novo PC, melhorar a qualidade das avaliações por pares realizadas. Caso esse processo não seja realizado adequadamente, os comentários produzidos pelos alunos poderão manter-se com a mesma proporção inicialmente encontrada no $\mathrm{PC} 1$, o que prejudicaria os pressupostos pedagógicos preconizados na seção 3 em relação aos objetivos de utilização da avaliação por pares na metodologia de ensino proposta.

\subsection{Análise dos Trabalhos Desenvolvidos}

Todas as plataformas de hardware desenvolvidas pelos alunos continham como dispositivos de entrada as chaves Liga/Desliga e botões do tipo push e, como dispositivos de saída, displays de 7-segmentos. Esses dispositivos citados são enunciados desde o início da disciplina como sendo obrigatórios. Por sua vez, demais dispositivos como módulos LCDs e Arduínos são considerados opcionais e não precisam necessariamente serem implementados para aprovação na disciplina. Vale ressaltar que, a implementação de dispositivos de Entrada/Saída (E/S) como módulos LCDs e Arduínos são de maior complexidade, exigindo um conhecimento adicional aos alunos sobre conceitos de E/S, microcontroladores e protocolos de comunicação.

Para investigar melhor essa situação, buscou-se identificar, cronologicamente entre os pontos de checagem, uma ligação ou relação entre as implementações dos subsistemas de E/S e o processo de avaliação por pares. Sendo assim, definiu-se como a existência de uma ligação ou relação entre um dispositivo opcional de E/S e a avaliação por pares os seguintes acontecimentos:

1) o aluno que está especificando ou implementando o dispositivo de E/S em sua plataforma de hardware foi avaliador, no ponto de checagem anterior, de um ou mais relatórios técnicos de alunos que descreviam esse dispositivo de E/S em suas plataformas de hardware; e

2) o aluno que está especificando ou implementando o dispositivo de E/S em sua plataforma de hardware recebeu no ponto de checagem anterior pelo menos uma ficha de avaliação onde consta, por meio da seção de comentários, uma recomendação para a utilização e implementação desse dispositivo de E/S.

Tomando como referência os módulos LCDs e para ilustrar cronologicamente a existência de relação entre a implementação de módulos LCDs nas plataformas de hardware e o processo de avaliação por pares, foi elaborado o gráfico da Figura 3. Nesse gráfico uma matriz $5 \times 5$ contendo 25 elementos é mostrada, representando os vinte e cinco projetos de toda a turma. A ideia é identificar nessa matriz os projetos que implementam os módulos LCDs em suas plataformas de hardware, buscando alocar projetos próximos uns dos outros (elementos adjacentes da matriz) quando houver a existência da ligação ou relação descrita no parágrafo anterior. Justamente por buscar mapear essa relação existente alocando projetos em elementos adjacentes, a matriz da Figura 3 foi denominada matriz de proximidade.

Iniciando no primeiro ponto de checagem (PC1), apenas em um único relatório o módulo LCD foi especificado como fazendo parte do subsistema de E/S de uma plataforma de hardware. Sendo assim, na posição central da matriz de proximidade (elemento $(3,3))$ da Figura 3, foi alocado esse único projeto que especificou o módulo LCD no relatório técnico durante o PC1. 

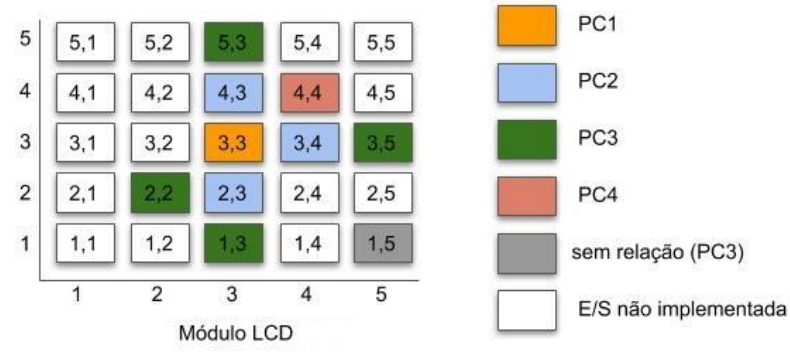

Fonte: os autores.

Figura 3 - Matriz de Proximidade levando em consideração o módulo LCD como dispositivo opcional de E/S implementado

Por sua vez, no segundo ponto de checagem (PC2), o módulo LCD foi descrito em outros três relatórios. Analisando o processo de avaliação colaborativa durante o PC1, percebeu-se que os alunos responsáveis por dois desses projetos foram avaliadores do relatório do projeto alocado no elemento $(3,3)$ da matriz, configurando-se, portanto, numa relação do tipo (1), como detalhado anteriormente. Quanto ao outro projeto, foi identificada a relação do tipo (2), onde o aluno do projeto alocado no elemento $(3,3)$ foi o avaliador e recomendou a implementação do módulo LCD por meio do preenchimento de sua ficha de avaliação. Sendo assim, devido à relação estabelecida mapearam-se, na matriz de proximidade, esses três novos projetos em elementos adjacentes ao projeto alocado no elemento $(3,3)$ da matriz.

Prosseguindo com a análise, durante o terceiro ponto de checagem (PC3), foram encontrados cinco novos projetos relacionados aos módulos LCDs em plataformas de hardware. Em quatro dos cinco projetos identificou-se a relação do tipo (1) ou do tipo (2), tendo sido devidamente mapeados na matriz de proximidade da Figura 3. O único projeto onde não se encontrou a relação do tipo (1) ou a relação do tipo (2) foi alocado no elemento $(1,5)$, não estando adjacente a nenhum outro projeto.

No quarto ponto de checagem (PC4), foi encontrado mais um projeto cujo relatório técnico descreveu a implementação do módulo LCD na plataforma de hardware. Por se tratar de relação do tipo (2) em que o aluno do projeto alocado no elemento $(4,3)$ foi o avaliador e recomendou a implementação do módulo LCD, o projeto foi mapeado no elemento adjacente $(4,4)$.

$\mathrm{O}$ mesmo procedimento de construção da matriz de proximidade também foi realizado para o dispositivo de E/S contendo Arduíno.

Por fim, no final da disciplina, os alunos identificados como tendo relação do tipo (1) ou (2) em relação ao Módulo LCD ou ao Arduíno foram individualmente convidados para uma entrevista sobre as decisões de projeto que eles tomaram em suas plataformas de hardware. Nessa entrevista, os alunos mencionaram, como fator de influência, o fato de terem lido sobre os dispositivos opcionais de E/S implementados em relatórios técnicos que foram avaliados por eles (relação do tipo (1)) ou por terem recebido, de seus avaliadores, sugestões de implementação em seus projetos desses dispositivos de E/S (relação do tipo (2)).

\section{Considerações Finais}

Neste artigo, buscou-se avaliar a aplicação das técnicas de avaliação por pares e de rubricas na promoção de interação entre alunos em práticas de ensino baseadas em problemas numa disciplina de graduação em Engenharia de Computação.

De acordo com os resultados apresentados na seção 4, a aplicação da metodologia descrita tem possibilitado aos alunos o acompanhamento dos diversos trabalhos e projetos 
que são desenvolvidos na disciplina; fomentado a interação entre os alunos, onde, por meio do preenchimento das fichas de avaliação, novos caminhos, alternativas, melhorias, adequações e ajustes podem ser efetivados e; estimulado o senso crítico e a capacidade de auto-avaliação ao permitirem que os alunos analisem os projetos desenvolvidos pelos seus pares.

Além disso, a avaliação por pares tem influenciado os alunos na implementação de dispositivos opcionais de E/S, motivando-os tanto na realização de trabalhos práticos adicionais de maior complexidade quanto no estudo de assuntos teóricos relacionados.

Por sua vez, as rubricas elaboradas de acordo com o apresentado na ficha de avaliação da Tabela 1, ao serem combinadas com o processo de avaliação por pares, têm facilitado e direcionado a produção de retornos formativos, servindo como guia dos processos de ensino e de aprendizagem e contribuindo para que os objetivos estabelecidos na disciplina sejam alcançados.

\section{Referências Bibliográficas}

ANDRADE, H. G. Using rubrics to promote thinking and learning. Educational Leadership, v. 57, n.5, p. 13-18, 2000.

ASIKAINEN, H.; VIRTANEN, V.; POSTAREFF, L.; HEINO, P. The validity and students' experiences of peer assessment in a large introductory class of gene technology. Studies in Educational Evaluation, v.43, p.197-205, 2014.

BARBOSA, M. W.; NELSON, M. A. V. Estamos prontos para utilizar a avaliação em pares na educação a distância? Um estudo de caso em um curso de especialização. Revista Novas Tecnologias na Educação (RENOTE), v.14, n.1, 2016.

BARROWS, H.; TAMBLYN, R. Problem-based learning: an approach to medical education. New York: Springer Publishing Company, 1 ed., 1980.

CAMPBELL, A. Application of ICT and rubrics to the assessment process where professional judgement is involved: The features of an emarking tool. Assessment and Evaluation in Higher Education, v.30, n.5, p.529-537, 2005.

FERREIRA, D. M.; SILVA, A. C. Avaliação de um web fórum por meio de rubricas. Meta: Avaliação, v.2, n.4, p.87-127, 2010.

FLORIAN-GAVIRIA, B.; GLAHN, C.; GESA, R. F. A software suite for efficient use of the European qualifications framework in online and blended courses. IEEE Transactions on Learning Technologies, v.6, n.3, p.283-296, 2013.

GAROUSI, V. Applying peer reviews in software engineering education: An experiment and lessons learned. IEEE Transactions on Education, v.53, n.2, p.182-193, 2010.

GIELEN, M.; WEVER, B. Structuring peer assessment: Comparing the impact of the degree of structure on peer feedback content. Computers in Human Behavior, v.52, p.315-325, 2015.

GONZÁLEZ-CHORDÁ, V. M.; MENA-TUDELA, D,; SALAS-MEDINA, P.; CERVERAGASCH, A.; ORTS-CORTÉS,I.; MACIÁ-SOLER, L. Assessment of bachelor's theses in a nursing degree with a rubrics system: Development and validation study. Nurse Education Today, v.37, p.103-107, 2016.

HUBA, M. E.; FREED, J. E. Learner-centered assessment on college campuses: Shifting the focus from teaching to learning. Pearson, 1 ed., 2000. 
LOBATO, A. S.; BRITO, S. R.; SOUZA, D. N. N.; FAVERO, E. L. Um sistema gerenciador de rubricas para apoiar a avaliação em ambientes de aprendizagem. In: XX SIMPÓSIO BRASILEIRO DE INFORMÁTICA NA EDUCAÇÃO. Florianópolis/SC, 2009.

MCGOLDRICK, K.; PETERSON, B. Using rubrics in economics. International Review of Economics Education, v.12, p.33-47, 2013.

NG, E. M. W. Using a mixed research method to evaluate the effectiveness of formative assessment in supporting student teachers' wiki authoring. Computers \& Education, v.73, p.141-148, 2014.

NARCISS, S. Feedback strategies for interactive learning tasks. In: SPECTOR, J. M.; MERRILL, M. D.; VAN MERRIËNBOER, J. J. G.; DRISCOLL, M. P. (Ed.). Handbook of research on educational communications and technology. Mahwah, NJ: Erlbaum, 3 ed., 2008, p.125143.

OLIVEIRA, T.; MARTINS, L. E. G.; STRINGHINI, D.; FAZENDA, A. L.; CAPPABIANCO. F. A. M. O ensino e aprendizado de Arquitetura e Organização de Computadores num currículo de engenharia de computação estruturado em uma abordagem prática e sistêmica. International Journal of Computer Architecture Education (IJCAE), v.6, n.1, p.2736,2017 .

PIEDRA, N.; CHICAIZA, J.; LÓPEZ, J.; ROMERO, A.; TOVAR, E. Measuring collaboration and creativity skills through rubrics - Experience from UTPL collaborative social networks course. In: EDUCATION ENGINEERING (IEEE EDUCON), 2010.

REDDY, Y. M.; ANDRADE, H. A review of rubric use in higher education. Assessment \& Evaluation in Higher Education, v.35, n.4, p.435-448, 2010.

SALES, A. B.; DEL, A. M.; SALES, M. B. Avaliação da aplicação da aprendizagem baseada em problemas na disciplina de interação humano e computador de curso de engenharia de software. Revista Novas Tecnologias na Educação (RENOTE), v.11, n.3, 2013.

SILVA, E.; MOREIRA, D. WebCoM: A tool to use peer review to improve student interaction. ACM Journal of Educational Resources in Computing, v.3, n.1, 2003.

TENÓRIO, T.; BITTENCOURT, I. I.; ISOTANI, S., SILVA, A. P. Does peer assessment in online learning environments work? A systematic review of the literature. Computers in Human Behavior, v.64, p.94-107, 2016.

TOPPING, K. Self and peer assessment in school and university: Reliability, validity and utility. In: SEGERS, M.; DOCHY, F. (Ed.). Optimising New Modes of Assessment: In Search of Qualities and Standards. New York, Boston, Dordrecht, London, Moscow: Kluwer Academic Publishers, 2003, p.55-87.

TRAHASCH, S. From peer assessment towards collaborative. In: ASEE/IEEE FRONTIERS IN EDUCATION CONFERENCE. Savannah - GA: ASEE/IEEE, 2004, p.16-20.

UGULINO, W.; MARQUES, A. M.; PIMENTEL, M.; SIQUEIRA, S. W. Avaliação colaborativa: Um estudo com a ferramenta moodle workshop. In: XX SIMPÓSIO BRASILEIRO DE INFORMÁTICA NA EDUCAÇÃO. Florianópolis/SC, 2009.

WANG, Y.; LI, H.; FENG, Y.; JIANG, Y.; LIU Y. Assessment of programming language learning based on peer code review model: Implementation and experience report. Computers \& Education, v.59, n.2, p.412-422, 2012. 